\title{
Nonverbal interaction between hitchhikers and drivers*
}

DAGMAR SCHMAUKS

\section{Abstract}

This essay reconstructs the implicit knowledge of a competent hitchhiker from the viewpoint of semiotics. The initial phase of hitchhiking is purely nonverbal. The hitchhiker starts the interaction by signaling that s/he wants a lift. In the standard case, the sign chosen is a culture-specific gesture. The driver produces one of the following four actions: (i) in the successful case, s/he stops and gives the hitchhiker a lift, whereas (ii) in the opposite case of ultimate non-success, s/he shows no perceptible reaction. In other constellations, s/he (iii) produces an 'answering' gesture or (iv) expresses a specific mood, intention, or attitude toward the hitchhiker by changing his or her driving behavior. This article concentrates on actions (iii) and (iv) and reconstructs them as equivalents of verbal utterances, each with a specific propositional content and illocutionary force. In the most elaborate interactions, one can detect a real 'gestural dialog' with several turn-takings.

\section{Introduction}

Hitchhiking is characterized by a genuine asymmetry between the actors. The hitchhiker wants to get a lift, and the driver is not at all socially obliged to fulfill this demand. This distinguishes the situation from face-to-face interactions between strangers - like asking for the time or for the way to a specific destination - where it would count as highly impolite to refuse help. It must be taken into account, however, that the general attitude toward hitchhiking depends on region and time. In remote areas with insufficient public transport, it counts as a normal behavior. If the area in question is very poor, hitchhiking may be so common that each hitchhiker has to pay for the trip, the price depending on distance or on difficulty of the road. On the other hand, recent criminal acts committed by drivers or hitchhikers dramatically decrease the inclination to ask for a lift or to offer one, respectively. 
From a semiotic point of view, hitchhiking can be reconstructed as a complex script the initial phase of which is necessarily purely nonverbal. This nonverbal interaction begins when the hitchhiker comes into the sight of the driver and signals from a distance that $\mathrm{s} / \mathrm{he}$ wants to get a lift. In the typology proposed by Ekman and Friesen (1969), this gesture belongs to the class of emblems. Emblems are produced intentionally, substitute a verbal utterance, have a fixed meaning, and are generally known within a culture.

In terms of speech act theory (cf. Searle 1969), the hitchhiker's gesture is successful if the driver stops and gives him or her a lift. In this case, a face-to-face communication starts in which speech and gestures are produced in parallel. A special case, namely the constellation $<$ female hitchhiker / male driver>, has been treated in detail in Schmauks 1997. This investigation covers the whole script of interaction from producing the hitchhiker's emblem to getting out of the car if the situation becomes threatening. Furthermore, it lists some practical, ecological, and social motives for hitchhiking.

The opposite case of ultimate non-success takes place if the driver shows no perceptible reaction - no gaze contact, no gesture, no slowing down. Here, the term 'interaction' comes to its limits, because it may be the case that the driver has not at all realized the hitchhiker. Intentional ignorance, however, is already an indexical sign - and a proof of Watzlawick's thesis (1967) that it is impossible not to communicate. An analogon in faceto-face interaction would be a question that does not trigger a verbal or nonverbal reaction.

Between these two extremes, however, one can locate a variety of driver's reactions, ranging from mere actions (like curiously slowing down) to a set of typical emblems. Frequently occurring examples are politely indicating a divergent goal and mockingly waving one's hand. The following sections propose a typology of the occurring (re)actions, based on their functions. The empirical basis or 'corpus' of this article (as well as for Schmauks 1997) is based on the author's own hitchhiking experiences in and outside Europe, collected in about 35 years and 35,000 kilometers.

Section 2 describes the strategies by which the hitchhiker 'prepares the stage' for getting a lift, mainly choosing the optimal place. Section 3 analyzes his/her subsequent task, namely choosing the right gesture and a promising addressee. The last two sections investigate the highly variable reactions of the non-stopping driver. One frequent possibility is again the production of emblems. Section 4 lists the most important variants and analyzes their forms and functions. All these emblems are well known from other contexts and therefore spontaneously understandable. Only here, we see a communication in the strict sense: The driver produces a specific 
gesture in order to transmit a specific meaning. Section 5 treats situations without such gestural communication in which, however, the driving behavior itself can be seen as an indexical sign. A suggestive example is the intentional variation of the distance between car and hitchhiker: Whereas going out of his or her way may be interpreted as an acceptance of the hitchhiker, passing by very close is interpreted as an explicit threat.

\section{Defining the framework of interaction}

The potential interaction begins as soon as the hitchhiker comes into the sight of the driver. The importance of 'nonverbal preliminaries' becomes clear by reconstructing them as equivalents of verbal utterances. Drivers must decide within seconds whether to stop. Absolutely central thus is a sophisticated choice of location, because it signals competence and sensitivity for the driver's needs. Seen from a functional point of view, the entire 'preparation of the stage' is thus a part of shaping one's image (cf. Goffman 1959).

The optimal site is visible from a distance (a requirement that restricts safe hitchhiking to the daylight), is flat, and offers at least one comfortable possibility for stopping. The desired direction should be unambiguous (no hitchhiking in front of a bifurcation!) and the traffic's average speed not too high. Hitchhiking immediately at a traffic light should be avoided because it can be interpreted as a too strong and too impolite request.

A second essential is the hitchhiker's outfit. Clothes, hair, makeup, baggage, etc., are always read as signs for specific features of their owner and become deciding factors in situations without verbal contact. Because the car is part of his or her private space, it is the driver who defines the rules of getting a lift. Clothing has to fit into the culture-specific codes (especially for women!) and should be clean enough (especially the shoes!). Every 'disguise' - like wearing reflective sunglasses, hats with broad brims, or hoods, etc. - is an easily understandable reason for nonstopping. The same is true for voluminous baggage and situations in which it remains open how many people want a lift.

\section{Emblems of the hitchhiker}

In order to be successful, the hitchhiker has to solve a three-fold task, the parts of which are investigated in the next sections. The analysis is a reconstruction of an otherwise implicit 'hitchhiking competence' and may be read as an advice as well. 


\subsection{Choosing the right emblem}

If one travels abroad, choosing the right emblem becomes essential. Very typical for natural languages as well as for speech-related gestures is (partial) synonymy - i.e., that one meaning can be transmitted by several signs. Well-known examples are the words 'film' and 'movie' (both referring to the same dynamic medium) and the culture-specific emblems of agreement ('yes' is gesturally expressed in central Europe by nodding, and in Bulgaria and Greece by shaking one's head). Taking such differences into account, it is not surprising that the request ' $I$ want a lift' also has several culture-specific codings.

A thorough investigation of the variants shows that they share some morphological features because the hitchhiker's general posture is fixed by sensomotoric demands. In order to monitor the traffic, s/he positions herself or himself at the edge of the road (eventually stepping somewhat into the road), facing approaching cars. This has the additional advantage that drivers are able to see the hitchhiker's face. In all variants I know, the arm is extended (often as far as possible), making the gesture optimally visible from a distance. Due to this requirement of visibility, one must use the right arm in countries where drivers drive on the right, and the left one in others. Shape and movement of the hand, however, are culture-specific. In central Europe, the hand forms a fist and the thumb is erected upward or in direction of the desired lift. Slight vertical movements may intensify this emblem.

Misunderstandings occur because, in other regions - for example, in eastern Mediterranean countries this emblem is used as an insult. Here, the thumb is interpreted as an erected penis and the movement specifies 'Sit on it!' In order to avoid serious problems, one should know the correct hitchhiker's emblem in these areas: extending the flat hand, palm downward, and slowly moving upward and downward. Also people from central Europe spontaneously understand this emblem because they know a closely related meaning, namely 'Slow down, please'.

\subsection{Choosing the right addressee}

A second task is choosing the right addressee. Trivially, people on dutypolice, ambulance, fire brigade and so on - should not be bothered by hitchhikers. Taxis are a different case: If they stop, they normally want the lift to be paid. As soon as one sees that all seats in a car are occupied, signaling becomes useless. In order to maximize one's own safety, one should not stop obviously inconsiderate or unsafe drivers - recognizable 
by exaggerated speed or zigzagging. Female hitchhikers are confronted with special problems and should therefore avoid cars with several men. And every hitchhiker should trust his or her own intuitions: Spontaneous aversion toward the driver (caused by whatsoever) is a clear signal not to enter the car.

\subsection{Choosing the right moment}

Choosing the right moment is equally important. One should start signaling early enough to give the driver a short time for deciding whether to stop. To avoid being a nuisance, one should stop signaling as soon as one realizes that the driver has not applied his or her brakes. A friendly but not provocative facial expression is most adequate for the situation in question.

If the driver does not stop, the hitchhiker should abstain from producing insulting emblems during or after the passing by of the car. S/he should take into account that the driver can see body movements for some time in the rear-view mirror, and that the revenge of an angry driver in a lonesome stretch of the road is not an advisable experience.

\section{Emblems of the driver}

Many non-stopping drivers are nevertheless motivated to communicate with the hitchhiker. The most frequent emblems - each of them with a specific propositional content and illocutionary force - are listed in the following sections.

\subsection{Informing about a divergent goal}

If the driver is in principle willing to offer a lift, but knows that s/he has to leave the main road soon, s/he can politely signal this fact to the hitchhiker by indicating the approaching action. The usual gesture in this situation is produced by positioning the flat hand vertically and moving it along the imaginary bend - thus iconically depicting the trajectory in the near future. The most comfortable variant is to indicate a turn to the left with the right hand and vice versa: This movement is physiologically most adequate and clearly visible from a distance. For optimizing visibility, the driver will show a right-angled bend even if the real turn (which is of no interest for the hitchhiker) has a different angle. The propositional 
content of this gestural utterance is 'I'll turn to the right (left) soon' and the illocutionary force is 'regretting'.

If there is time enough, the hitchhiker can react with a third emblem. In this case, a real 'gesture dialog' with two turn-takings takes place. A smile of resignation, eventually accompanied by shrugging one's shoulders or (in an attitude of helplessness) lifting both hands, has the propositional content of 'Okay, I understand' and the illocutionary force 'regret' as well. Another friendly possibility is waving one's hand ('Have a good trip!'). This mutual expression of regret has a primarily phatic function: It creates a cooperative atmosphere between two road users, although they will not come into closer contact.

\subsection{Giving another reason for not stopping}

Functionally very similar are the emblems by which the driver gives another reason for not stopping. A frequent case - especially in rural areas where cars are means for transporting animals and goods as well is a completely occupied car where no place is left for the hitchhiker. Shrugging one's shoulders or pointing to the fully occupied back seat are easily understandable. A related case is pointing to a huge dog in the car. Also this can be verbalized as 'Sorry, there is no place for you', even if the utterance meaning is something more specific like 'Sorry, but my dog doesn't accept strangers'.

As in section 4.1, the illocutionary force is 'regret' and the hitchhiker can react by the same emblems listed there. Trivially, a highly frustrated hitchhiker can refuse to show mutual understanding and may prefer to produce insulting emblems. This reaction is not only potentially dangerous (cf. section 3.3) but also reduces the driver's general willingness to accept hitchhikers in the future. As a sign of solidarity between hitchhikers (who are a kind of 'community' although they may never meet one another), one should reinforce every positive reaction of drivers - at least by a smile.

\subsection{Criticizing the hitchhiker}

Whereas the last two sections treated emblems with a clearly positive meaning, the emblems analyzed now express a criticism.

If the hitchhiker has chosen an unfavorable site without possibilities for safe stopping, the driver can shrug his or her shoulders while passing by, eventually accompanied by a 'questioning' facial expression. The propositional content is the statement 'I can't stop here' or as a generalized 
prediction 'Nobody will stop here'. The illocutionary force is 'criticism' or stronger 'reproach'.

Even here, we see a kind of cooperation, because one can reconstruct the transmitted meaning as 'I would have given you a lift, but your (stupid) choice of site has made stopping impossible'. If the hitchhiker realizes the reason for the driver's non-stopping, a rational reaction would be to choose a more suitable location immediately.

A more explicit variant of criticism is an emblem of 'chasing' the hitchhiker toward the edge of the road. The form is well known for scaring off approaching animals (and therefore quite impolite for addressing humans): the flat right hand, positioned vertically, is bent and stretched again several times. The sentence meaning is 'I hereby request you to step aside'; the emblem can be verbalized - depending on the driver's mood also as 'Move along' or stronger as 'Piss off!' Only if the hitchhiker is able to see the driver's facial expression, s/he can decide whether the emblem is an advice ('You step too far into the road'), a request ('Don't hamper me!'), an expression of annoyance ('What are you doing here?'), or even a threat ('Out of my way, or I crust you!'). This emblem can be intensified by angry hooting.

Here, the only advisable reaction is stepping aside. On the one hand, this signals understanding of the driver's emblem; on the other hand, it avoids an escalation of the interaction (cf. section 5.4).

\subsection{Mocking the hitchhiker}

Mocking the hitchhiker is another variant of uncooperative communication. Simply waving one's hand may be a harmless kind of teasing, meaning something like 'Good luck with others!' The driver should take into account, however, that a frustrated hitchhiker (e.g., having been on the road for hours and in bad weather) is probably not in the mood for mockery.

All well-known emblems of insulting are produced in this context as well: showing one's tongue, making a long nose, and showing the extended middle finger. They all have the propositional content 'That's what you think!' or 'No way!' The illocutionary force ranges from teasing to more severe forms of mockery.

Showing one's fist with thumb extended downward is a clear sign of rejection as well. In ancient gladiator fights, this emblem had the meaning 'Kill him!' Here, it may be verbalized as 'Down with all hitchhikers!'

An experience of my own shows that this emblem may be produced by hitchhikers as well. When I waited for a lift, a driver passed by, showing me the extended thumb directed downward. Some minutes later, another 
driver gave me a lift. As he had a much faster car, we overtook the first car soon. When its driver arrived at my new location, I recognized the car and now it was I who could present the same emblem - sweet revenge! (Of course, he again did not stop.)

\section{Driving behavior as an indexical sign}

In the situations listed in this section, the driver does not produce an emblem or another intentional nonverbal sign. The visible driving behavior, however, can be interpreted as an indexical sign of the driver's mood, intention, or attitude toward the hitchhiker (or hitchhikers in general). For similar cases in the context of traffic, cf. von Savigny 1995.

\subsection{Scrutinizing the hitchhiker}

In order to decide whether to stop, the driver has at first only one knowledge source, namely the visual impression of the hitchhiker. A thorough scrutinizing needs some time and thus a slowing down of the car. This mere action can therefore be interpreted as indication that the driver starts a decision process and is in principle willing to offer a lift.

If the test fails, the whole visible driving action has three steps: slowing down, scrutinizing the hitchhiker, and accelerating again. In this case, the hitchhiker can suspect that something is wrong with him or her: unsuitable location? wrong appearance? too young? S/he should take into account, however, that there is a second motive for intensive scrutinizing, namely non-specific curiosity. Here, the slowing down can be verbalized as 'Let's have a close look at that guy' or 'Who can that be-hitchhiking today - where everyone has a car?'

The hitchhiker must accept the driver's legitimate desire of scrutinizing before stopping, but there is no need for changing his/her own behavior. A moderate smile is adequate, but exaggerated smiling as well as additional waving or jumping is already too much effort and may result in a negative response. The driver's line of argument could be 'If somebody is struggling so much for a lift, what is wrong with him or her?'

\subsection{Accepting the hitchhiker}

In many cases, the driver accepts the hitchhiker in principle, but has personal reasons for not stopping. Maybe $s /$ he never stops, because s/he 
has had unpleasant experiences in the past. Highly stressed people can regard their car as a precious room for staying comfortably alone. Other reasons are only temporary: The driver is in a hurry, prepares a talk, wants to make some telephone calls, or simply is not in the mood for communicating.

In order to tell the hitchhiker that non-stopping does not mean nonacceptance, the driver can simply enlarge the distance between car and hitchhiker. This driving around the hitchhiker at a comfortable distance has three positive effects: $\mathrm{S} / \mathrm{he}$ is not physically threatened, feels respected, and the next car has a better view to him or her. Trivially, this strategy is not possible on small roads with heavy traffic. The sentence meaning is 'I don't stop, but I give you space for reaching your own goals'. The underlying attitude is acceptance of the hitchhiker's goals. Furthermore, the behavior is an expression of politeness.

\subsection{Mocking the hitchhiker}

Functionally very similar to the emblems discussed in section 4.4, the driving behavior itself can express a mockery. A typical example is emphasized accelerating immediately in front of the hitchhiker, as well as loud and long hooting without any practical reason. The sentence meaning may be something like 'Step aside'. If this ordering behavior is produced with the expression of disrespect and disdain, an adequate verbalization would be an insulting utterance such as 'Step aside, you fool!'

This kind of behavior is exclusively produced by very young male drivers who want to impress their friends who sit in the same car or in the following car. In ethological terms, these actions are part of the so-called 'display behavior'.

\subsection{Threatening the hitchhiker}

In contrast with the signs of acceptance (cf. section 5.2), the driver can also reduce the distance between car and hitchhiker. In moderate cases, this movement is an actional equivalent of the 'chasing' emblems, verbalized in section 4.3 as 'Get out of my way!' Even more aggressive are drivers racing through puddles and intentionally splashing the hitchhiker. The sentence meaning is something like 'The street is the territory of the drivers!' This action has a strong expressive function; it is a result of aggression, hatred, or enmity. 
If the car comes very close, however, the action becomes a severe physical threatening, which forces the hitchhiker to step (or in the extreme case, to jump) to the edge of the road. This example shows that there exists a gradual transition from

- an indexical sign (informing about the disrespect of the driver) to

- the gestural equivalent of a threat ('Get out of my way!'), and finally

- to the attempt to really injure (or even to kill) the hitchhiker.

\section{Note}

* This article is dedicated to the numerous friendly drivers who gave me lifts, talks, and advices, who shared their coffee and sandwiches with me, and who enlarged my knowledge about diverse topics from apiculture to the signs of the zodiac. Thanks are due to Roland Posner for helpful comments on a previous version of the text.

\section{References}

Ekman, Paul and Wallace V. Friesen (1969). The repertoire of nonverbal behavior: Categories, origins, and coding. Semiotica 1: 49-98.

Goffman, Erving (1959): The Presentation of Self in Everyday Life. Garden City, NY: Doubleday. (German translation: Wir alle spielen Theater. Die Selbstdarstellung im Alltag. Munich: Piper 1969. 6th edition 1997.)

Savigny, Eike von (1995). Autofahrerzeichen: Funktion, System, Autonomie. Zeitschrift für Semiotik 17: 105-128.

Schmauks, Dagmar (1997). Dialoge zwischen Anhalterinnen und Autofahrern. Rekonstruktion und Ratschläge. Psychosozial 69: 87-95.

Searle, John R. (1969). Speech Acts. Cambridge: Cambridge University Press. (German translation: Sprechakte. Frankfurt a.M.: Suhrkamp 1971.)

Watzlawick, Paul; Beavin, Janet H.; and Jackson, Don D. (1967). Pragmatics of Human Communication. A Study of Interactional Patterns, Pathologies, and Paradoxes. New York: Norton. (German translation: Menschliche Kommunikation. Formen, Störungen, Paradoxien. Bern: Huber 1969.)

Dagmar Schmauks (b. 1950) is Privatdozentin at Arbeitsstelle für Semiotik in Berlin <dagmar.schmauks@TU-Berlin.de>. Her principal research interests are orientation in space, maps, and bodily sign systems. Her major publications include Orientierung im Raum. Zeichen für die Fortbewegung (2002), Landkarten als synoptisches Medium (1998, edited with Winfried Nöth), and Multimediale Informationspräsentation am Beispiel von Wetterberichten. Eine semiotische Analyse (1996). 